International Journal of Engineering \& Technology, 7 (2.31) (2018) 29-33
International Journal of Engineering \& Technology
SPC
Website: www.sciencepubco.com/index.php/IJET
Research paper

\title{
Color detection in RGB-modeled images using MAT LAB
}

\author{
P. Sudharshan Duth ${ }^{1 *}$, M. Mary Deepa ${ }^{2}$ \\ ${ }^{1}$ Dept. of Computer Science, Amrita School of Arts and Sciences, Amrita Vishwa Vidyapeetham, Mysuru, Karnataka, India. \\ ${ }^{2}$ Dept. of Computer Science, Amrita School of Arts and Sciences, Amrita Vishwa Vidyapeetham, Mysuru, Karnataka, India.. \\ *Corresponding author E-mail:sudhisp@gmail.com
}

\begin{abstract}
This research work introduces a method of using color thresholds to identify two-dimensional images in MATLAB using the RGB Color model to recognize the Color preferred by the user in the picture. Methodologies including image color detection convert a 3-D RGB Image into a Gray-scale Image, at that point subtract the two pictures to obtain a 2-D black-and-white picture, filtering the noise picture elements using a median filter, detecting with a connected component mark digital pictures in the connected area and utilize the bounding box and its properties to calculate the metric for every marking area. In addition, the shade of the picture element is identified by examining the RGB value of every picture element present in the picture. Color Detection algorithm is executed utilizing the MATLAB Picture handling Toolkit. The result of this implementation can be used in as a bit of security applications such as spy robots, object tracking, Color-based object isolation, and intrusion detection.
\end{abstract}

Keywords: MATLAB, picture handling tool kit, shading location, RGB picture, picture division, picture separating, bounding box.

\section{Introduction}

Shading could be a standout among the foremost basic highlights of a Picture. If the shade of the live video or the progressive image is detected, then the results of this detection can be used in various scientific applications. Shading review is a necessary advance in numerous laptop vision frameworks. This work utilizes the picture in MATLAB to visualize a selected shading in a specific image. The Picture handling tool kit provides various reference checks, techniques, and applications for picture handling, detection and division. MATLAB based picture handling may be a unthinkably pleasing additionally, frequently used image primarily based computation to implement Picture based algorithms[1][2][3]. The picture is a Matrix of picture element values. MATLAB takes each answer as a Matrix, that makes it the foremost overall used platform for image Processing. Picture will address gray-scale, RGB, HSV, and alternative shading models. The RGB demonstrate is utilized here to examine the shading within the picture[4] [5]. The RGB demonstrate could be a shading model that joins red, green and blue lights in numerous approaches to manage create a mix of tones. In the RGB show, the picture incorporates a meeting of $\mathrm{MxNx} 3$ pixels for $m$ lines and $n$ domains about pixels to those red, green, What's more blue segments of the photo.

The RGB appear employments eight odds with handle those red, green Furthermore blue segments of the picture..Since the images are attended as frameworks within the RGB demonstrate coherent operations on the images is related with specific shades within the picture. The Picture handling toolkit is a MATLAB toolkit that provides a wide range for image modification, examination, dissent segment, shading acknowledgment, shading modification, to mention the terribly least. It as well offers a mixture of help image forms, together with high stream, imaging and gig a-pixel assurance.

\section{Literature survey}

"N. Otsu(1979) [1] in his paper proposed a technique to pick out a threshold mechanically from a grey level bar graph has been derived from the point of view of discriminant analysis. This directly deals with the matter of evaluating the goodness of thresholds. associate optimum threshold (or set of thresholds) is chosen by the discriminant criterion; specifically, by maximising the discriminant measure alphabetic character (or resultant the measure of disjunction of the categories in grey levels). G.Wyszecki., et al(1982) [2] proposed a research work that describes regarding the color science ideas and strategies. The RGB show is used here to acknowledge the shading within the image. The RGB show could be a shading model that joins red, inexperienced associated blue lights in numerous approaches to create an assortment of hues. Brownrigg, D. R. K(1984) [3] in his paper mentioned regarding the actual cases of filters and needs. The notion of a lowest weighted median filter, of a taxonomic group that act identically is introduced. J.Kittler.,et al (1986) [4] in his paper proposed a research work that provides the efficient answer for the matter of minimum error thresholding that comes below the idea of object and picture element level grey values being ordinarily distributed. This is applicable for mutithreshold choice. R. C. Gonzalez.,et al (1992) [5] in his paper Digital Image process discusses Image segmentation subdivides a picture into its constituent regions or objects. The amount of segmentation depends on the matter to be solved. Non-trivial image segmentation is one among the foremost tough tasks in image process. The accuracy of the segmentation determines the final word success or failure of a computerised analysis program. N. R. Pal., et al (1992) [6] in his paper mentioned the constraints of a number of the prevailing threshold choice techniques. The approximate minimum error thresholding algorithmic rule of Kittler and Illingworth has been changed considering a Poisson distribution for the gray level rather than the normally used 
distribution. The modified technique is found to be far better from the purpose of view of each convergence and segmental output. J.L. Vincent(1993) [7] in his paper describes regarding the morophological reconstruction. The quality parallel and ordered approaches to reconstruction are concisely recalled; their common disadvantage is their inefficiency on standard computers. to boost this case, a brand new algorithmic rule is introduced, that relies on the notion of regional maxima and makes use of breadth initial image scanning's enforced via a queue of pixels. Solihin, Y.,et al (2000) [8] in his paper discuss the advantages of a multi-stage thresholding approach that thresholds gray scale images stage-bystage. The ability to use numerous data from the image to help the assignment of a intensity level is central to the multi-stage approach. therefore it's better suited to pictures that have consistent characteristics as encountered in specific applications. R. S. Berns(2002) [9] in his paper describes that MATLAB takes every answer as a network, that makes it the foremost usually used image making ready stage. photos will speak to grey scale, RGB, HSV, and alternative shading models. Gonzalez woods \&amp; Eddins., et al (2004) [10] in his paper Digital image process using Matlab describes ways for detection shapes and color of objects which has been introduced antecedently. It tells relating to conversion of RGB image to gray scale image and so to black and white (binary) image and much additional, but the study of form recognition don't seem to be represented. Alasdair McAndrew (2004) [11] in his paper provides an in depth introduction to Digital image process with Matlab.M. Sezgin.,et al(2004) [12] In his work, he conducted an intensive study of image threshold ways, categorizing them, presenting their formulas in a consistent notation, and eventually comparing performance. the threshold technique is classified by bar graph form, spatial cluster, entropy, object attribute, spatial correlation, and native grayscale surface. It additionally identifies higher threshold algorithms than NDT and document image applications. Abadpour, A.et al (2005) [13]in his paper color image process using principal element analysis describes the Color recognition involves comparison of every pixel within the metric and leads to the dominant color because the color of the given object are explained. Senthamaraikannan, D.et al (2014) [14]in his paper real time Color recognition proposes new real time color recognition features, i.e., extracting primary colours for the aim of vision-based human-computer interaction. Vision-based human-computer interaction can be achieved by analyzing segmental primary color regions primarily focused on color-based image segmentation and vision primarily based color recognition by addressing these difficulties. However, cluttered backgrounds, unknown lighting conditions and multiple moving objects create this tasks difficult. Rani, N., et al(2015[15] in her research work mainly focus on the development of an automatic number plate extraction and recognition algorithm by incorporating constructs like edge detection, horizontal and vertical edge processing using fixed threshold technique. The extracted number plate region is again processed using template matching algorithm for the recognition of the characters embossed on the number plate with respect to every individual piece of number plate. The algorithm developed has achieved an accuracy of around $100 \%$ and works for both front and rear images of the car. Rani, N.,et al(2016)[16]In research work mainly focus on evaluating the performance of various feature extraction techniques with respect to Telugu character recognition systems and analyze its efficiencies and accuracies in recognition of Telugu character set".

\section{Methodology}

The picture consists of many pixels. every picture element corresponds to a code. The total of those codes provides a whole image. After analyzing the code, the code may be used for the definition of Colors, and therefore the Colors of those definitions are used for the picture element markers used to determine the Colors within the image.

\section{Algorithm for Color Detection in Images}

Step 1: Read an Input Image.

Step 2: Creating Red, green and blue Color bands of the Image.

Step 3: Compute and plot the Red, Green, Blue Color band Histogram.

Step 4 :Convert Input Image to gray-scale image.

Step 5:Track BLUE entity in real-time, we have to subtract the BLUE segment from the gray scale picture to remove the red segments in the picture.

Step 6: Apply Median-filter to remove noise.

Step 7:Transform the gray-scale picture into a black and white Picture.

Step 8:Extract every pixels less than 300px.

Step 9:Select all the associated segments in the picture.

Step 10:Find Centroid, Area, Bounding Box using Region props.

Step 10.1: Apply rectangular box for each Blue color in Image. Step 11: Stop

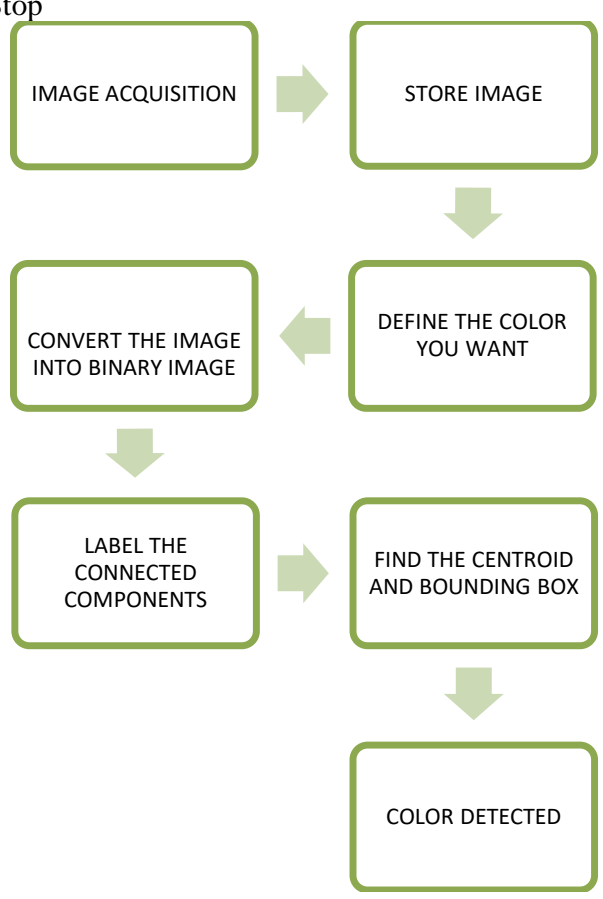

Fig. 1: Flow Chart of algorithm

\section{Implementation}

\section{Read the input image}

The first step in an image processing task is to have a picture to recognize the color. People can take it from the camera or load previously clicked images from memory. If the resolution of the image is $\mathrm{MxN}$, the RGB format of the image is a threedimensional matrix of size $\mathrm{MxNx} 3$, where each dimension of RGB is an RGB format representing a color picture in the most common format. The input image matrix represents the red, green, and blue segments of the picture.

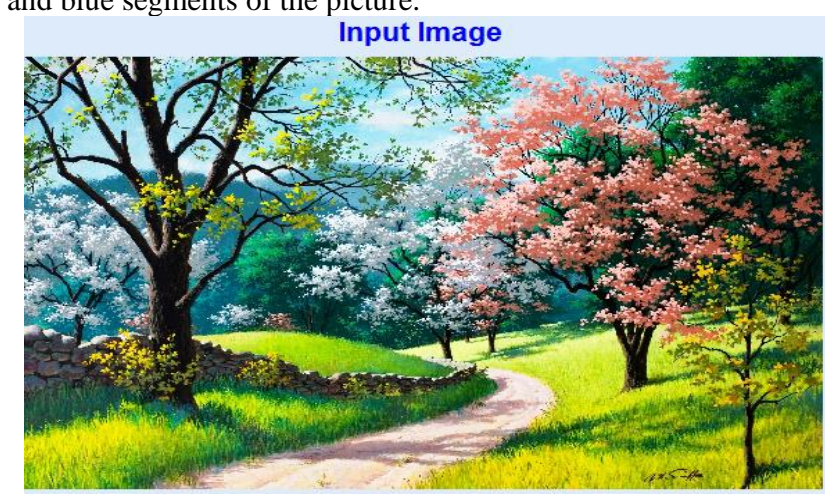

Fig. 2: Input image 
2. Generating red, green and blue color bands of the image

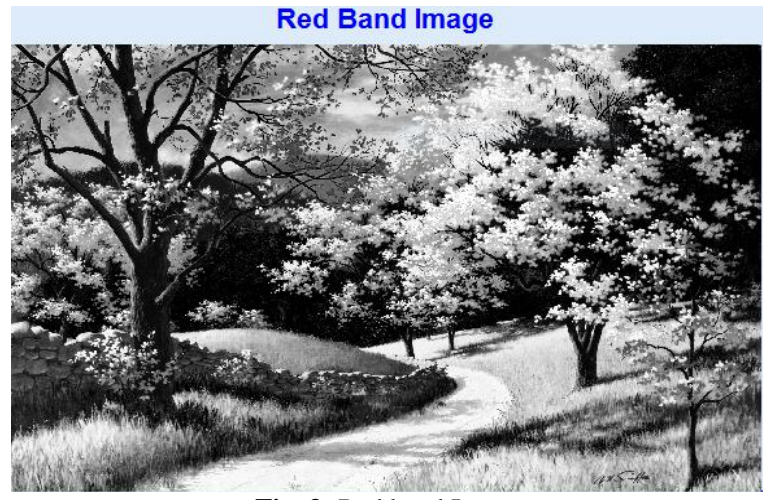

Fig. 3: Red band Image

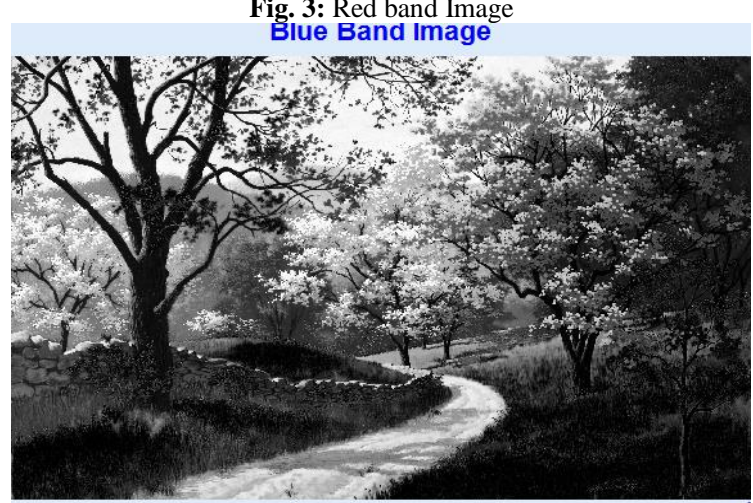

Fig. 4: Blue band Image

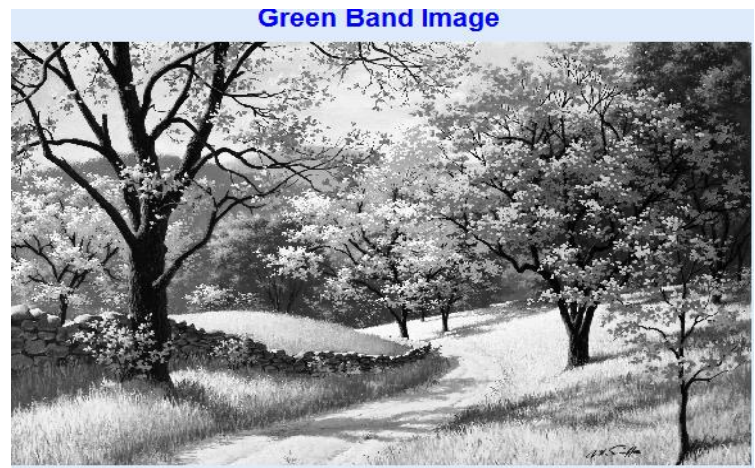

Fig. 5: Green band Image

The red, green, and blue bands are extracted from the input image into three independent two-dimensional matrices, for each color part. The first, second and third dimensional matrix of the RGB image each contain the red, green and blue components.

\section{Plotting of histogram}

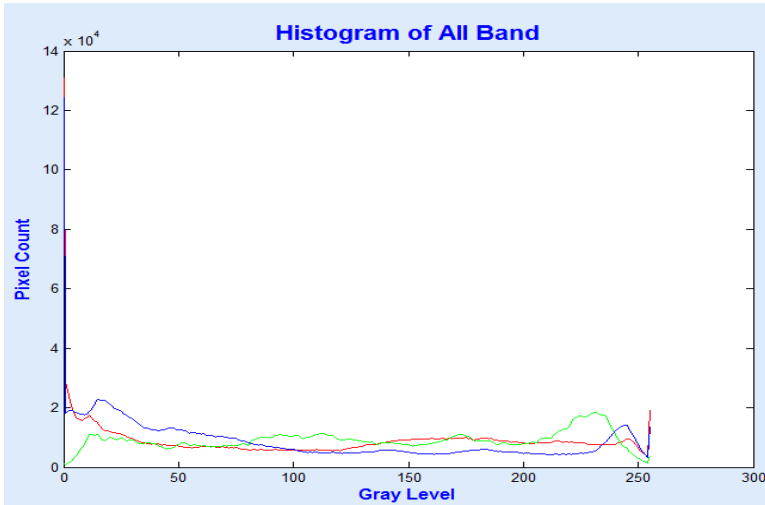

Fig. 6: Histograms of all bands

In photography and image processing, the histogram is the color distribution in the image. Use MATLAB's imhist () function to calculate and plot the histograms for the red, green, and blue bands.

\section{Convert input image to GRAY-Scale image}

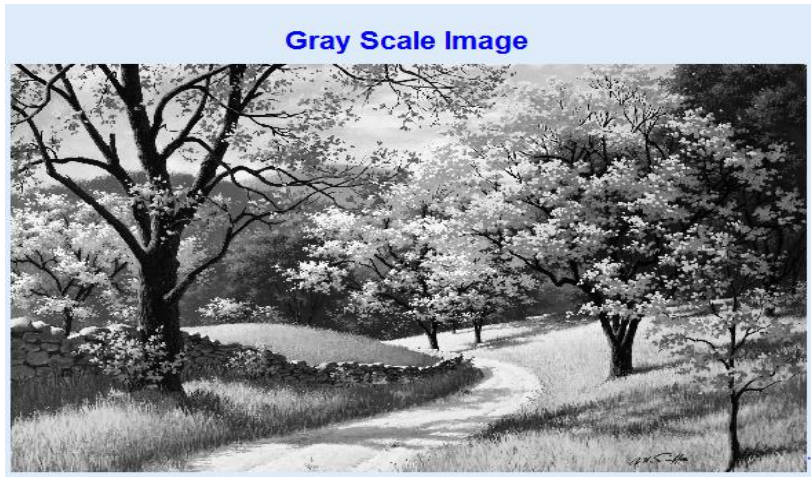

Fig. 7: Gray scale Image

The RGB picture is regenerate to a grayscale picture[6], and therefore the grayscale format of the image may be a twodimensional image containing the intensity value of every picture element of the image. Often, grayscale pictures increase process speed by changing three-dimensional pictures to two-dimensional pictures, area unit simple to ascertain and cut back the quality of the code, leading to a reduction within the range of bits used to represent every picture element of the image.

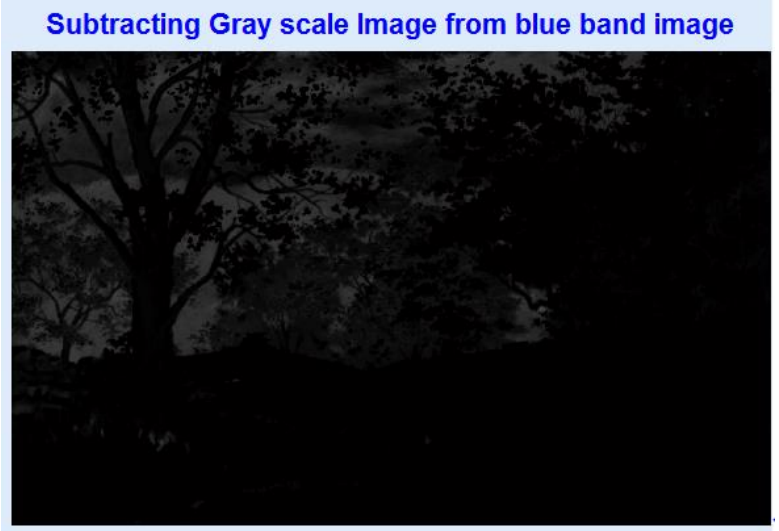

Fig. 8: Subtracting gray scale from blue band Image

The gray-scale Image is then subtracted from the Blue zone Image to capture the blue colour in the image. Similarly, the grayscale pictures are subtracted from the red and green bands to discover red and green. The grayscale image is subtracted from the blue band of the input image to extract the blue a part of the image.

\section{Median filtering}

Median filtering is employed to get rid of unwanted noise from the image whereas protective the originality of the image [15] [16]. The image is regenerate into grayscale image.To this extracted image median filter is applied so as to get rid of the noise within the image. throughout this method, the picture element value is regenerate to the median of the $3 \times 3$ sampling window, whereas the picture element worth remains unchanged at the sampling window boundary. 


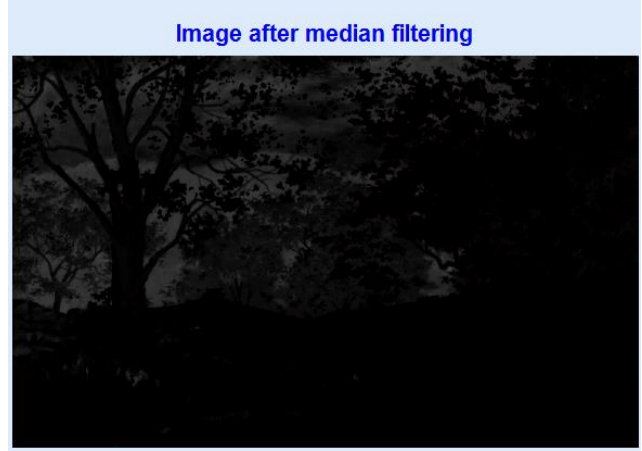

Fig. 9: Image after median filtering

6. Transform the gray-scale picture into a black and white

\section{Picture}

This filtered picture is then changed to a black and white picture that works only in the region of interest ( 1 for yes, 0 for no). The binary image is generated [7] [8] [10] from the filtered image using a threshold process. In threshold, every picture element of the image is assigned 1 or 0 , which is assigned "1" (white) if the picture element value is greater than the set threshold, and "0" (black) otherwise. Use the intensity histogram of the picture to calculate the threshold of the picture[11] [12] [13] .

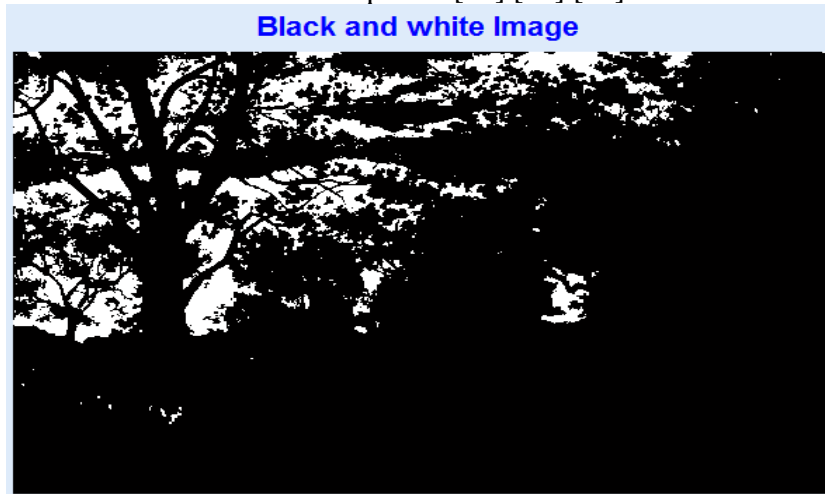

Fig. 10: Black and white image

\section{Extract every pixels less than 300px}

Remove every objects with a color of less than 300 pixels from the image.

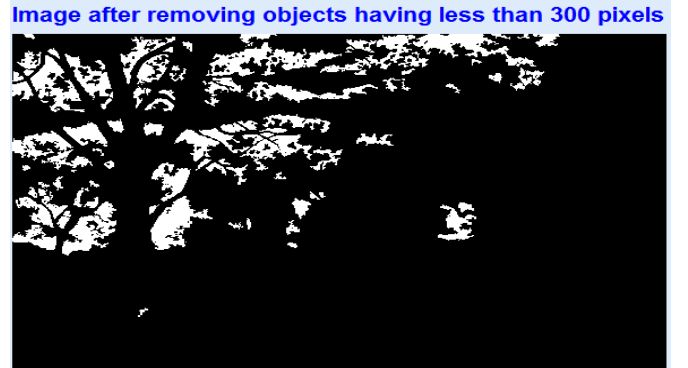

Fig. 11: Image after removing objects having less than 300 pixels

\section{Recognizing the boundaries of an object}

3D input images were converted to 2D arrays. Now select one of the random pixels in the background of the object and move it clockwise or counter clockwise to get other pixels. This forms the boundaries of the picture. Here the boundaries of the object are searched (in 8 chaining pixels).

\section{Bounding box of the colored object}

The detected object is indicated by a hypothetical rectangle with its sides parallel to the axis.

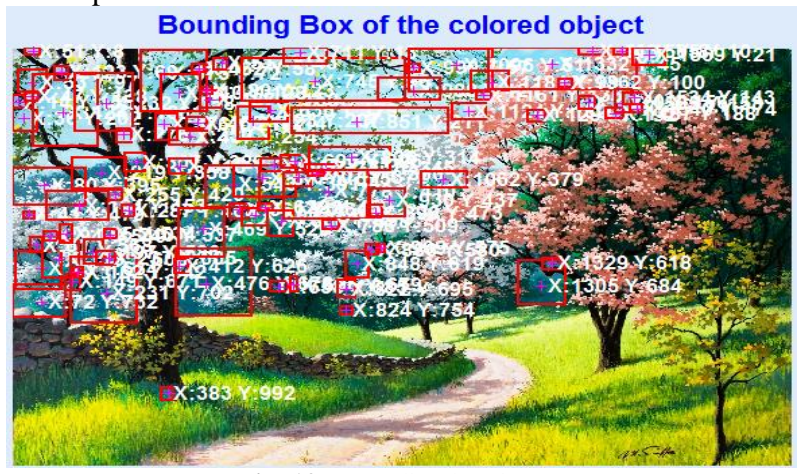

Fig. 12: Bounding box image

\section{Result}

The results of this detection depends on the threshold set for the image. The most drawback with the threshold is that solely the intensity values of the picture element are taken under consideration and no relationship between them is taken into account. generally further pixels are detected that don't seem to be a part of the specified space, and these errors increase with increasing noise. There is a problem within the captioning technique of associate associated element for image segmentation that associate overlapping object is treated as simply associate object if it exists within the image.

\section{Conclusion}

In this research work ,using MATLAB-2014a Image Processing Toolbox, we detect colors of two-dimensional images using RGB Model with color thresholds to detect red, blue, green, magenta, yellow and cyan. In addition to this we also detect colored objects in the limited region by using center of gravity of the selected region. The results seems to be improved and it is tested on a set of varied colored images with various intensities. The Results are found to be efficient and proximity.

\section{References}

[1] Otsu N, "A threshold selection method from gray-level histograms", IEEE transactions on systems, man, and cybernetics, Vol.9, No.1, (1979), pp.62-66.

[2] Wyszecki G \& Stiles WS, Color science, New York: Wiley, (1982).

[3] Brownrigg DRK, "The weighted median filter", Communications of the ACM, Vol.27, No.8,(1984), pp.807-818.

[4] Kittler J \& Illingworth J, "Minimum error thresholding", Pattern recognition, Vol.19, No.1, (1986), pp.41-47.

[5] Gonzalez RC \& woods RE, Digital Image Processing, AddisonWesely Publishing Company, (1992).

[6] Pal NR. \& Bhandari D, "On object background classification", International journal of systems science, Vol.23, No.11, (1992), pp.1903-1920.

[7] Vincent L, "Morphological grayscale reconstruction in image analysis: applications and efficient algorithms", IEEE transactions on image processing, Vol.2, No.2, (1993), pp.176-201.

[8] Solihin, Y \& Leedham CG, "The multi-stage approach to greyscale image thresholding for specific applications", Nanyang Technological University, School of Computer Engineering, Nanyang Avenue, Republic of Singapore, (2000).

[9] Berns, RS \& Reiman DM, "Color managing the third edition of Billmeyer and Saltzman's Principles of Color Technology", Color Research \& Application, Vol.27, No.5,(2002), pp.360-373.

[10] Gonzalez RC, Woods RE \& Eddins S.L, Digital Image Processing Using MATLAB, Pearson Education, Inc, (2004).

[11] McAndrew A, "An introduction to digital image processing with matlab notes for scm 2511 image processing", School of Computer Science and Mathematics, Victoria University of Technology, (2004). 
[12] Sezgin M \& Sankur B, "Survey over image thresholding techniques and quantitative performance evaluation", Journal of Electronic imaging, Vol.13, No.1,(2004), pp.146-166.

[13] Abadpour A, Color Image Processing Using Principal, (2005).

[14] Senthamaraikannan D, Shriram S \& William J, "Real time color recognition", International Journal of Innovative Research In Electrical, Electronics, Instrumentation And Control Engineering, Vol.2, No.3,(2014).

[15] Rani NS, Neethu OP \& Ponnath N, "Automatic Vehicle Tracking System Based on Fixed Thresholding and Histogram Based Edge Processing", International Journal of Electrical and Computer Engineering, Vol.5, No.4,(2015)

[16] Rani NS, Verma SK \& Joseph A, "A Zone Based Approach for Classification and Recognition Of Telugu Handwritten Characters", International Journal of Electrical and Computer Engineering, Vol.6, No.4,(2016). 